\title{
A novel score to estimate the risk of pneumonia after cardiac surgery
}

\author{
Arman Kilic, MD, Rika Ohkuma, MD, Joshua C. Grimm, MD, J. Trent Magruder, MD, Marc Sussman, MD,
} Eric B. Schneider, PhD, and Glenn J. R. Whitman, MD

\section{ABSTRACT}

Objective: The purpose of this study was to derive and validate a risk score for pneumonia (PNA) after cardiac surgery.

Methods: Adults undergoing cardiac surgery between 2005 and 2012 were identified in a single-institution database. The primary outcome was postoperative PNA. Patients were randomly assigned to training and validation sets in a 3:1 ratio. A multivariable model was constructed incorporating univariate pre- and intraoperative predictors of PNA in the training set. Points were assigned to significant risk factors in the multivariable model based on their associated regression coefficients.

Results: A total of 6222 patients were included. The overall rate of postoperative PNA was 4.5\% $(\mathrm{n}=282)$. A 33-point score incorporating 6 risk factors (age, chronic lung disease, peripheral vascular disease, cardiopulmonary bypass time, intraoperative red blood cell transfusion, and pre- or intraoperative intra-aortic balloon pump) was generated. The model used to generate the score in the training set was robust in predicting PNA $(\mathrm{c}=0.72, P<.001)$. Predicted rates of PNA increased exponentially with increasing risk score, ranging from $1.2 \%$ $($ score $=0)$ to $59 \%($ score $=33)$. There was significant correlation between predicted rates of PNA based on the training cohort and actual rates of pneumonia in the validation cohort in weighted regression analysis $(r=0.74, P<.001)$. The composite score outperformed the STS prolonged ventilation model in predicting PNA in the validation cohort (c-index 0.76 vs 0.71 , respectively).

Conclusions: This 33-point risk score is strongly predictive of postoperative PNA after cardiac surgery. The composite score has utility in tailoring perioperative management and in targeting diagnostic and preventative interventions. ( $\mathrm{J}$ Thorac Cardiovasc Surg 2016;151:1415-21)

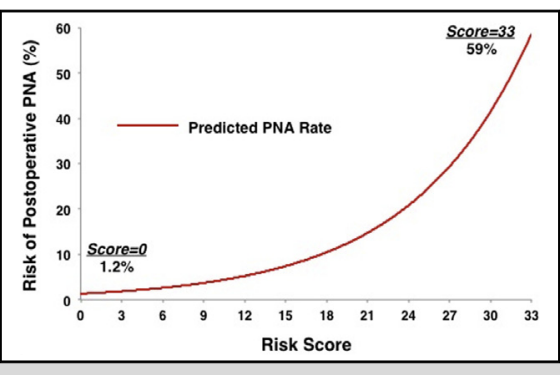

Predicted rates of postoperative pneumonia by risk score.

Central Message

We constructed a 33-point risk score for postoperative pneumonia after cardiac surgery incorporating 6 significant variables.

\section{Perspective}

We derived and validated a 33-point risk score for postoperative pneumonia after cardiac surgery that has potential utility in risk modification, directing diagnostic and preventative measures, and in clinical research.

See Editorial Commentary page 1422.
Pneumonia is the most common nosocomial infection after cardiac surgery. ${ }^{1}$ Its occurrence in the postoperative setting after heart surgery has been associated with an increased risk of mortality, readmission, and prolonged intensive care unit and hospital stay. ${ }^{1-3}$ Not unexpectedly, therefore, and perhaps as a consequence of its adverse impact on these outcomes, postoperative pneumonia also is associated with significantly greater costs of care. ${ }^{3,4}$ Risk

From the Division of Cardiac Surgery, Department of Surgery, The Johns Hopkins Medical Institutions, Baltimore, Md.

Read at the 95th Annual Meeting of The American Association for Thoracic Surgery, Seattle, Washington, April 27, 2015.

Received for publication April 27, 2015; revisions received Nov 28, 2015; accepted for publication Dec 13, 2015.

Address for reprints: Glenn J. R. Whitman, MD, Division of Cardiac Surgery, Johns Hopkins Hospital, 1800 Orleans St, Sheikh Zayed Tower Suite 7107, Baltimore, MD 21287 (E-mail: gwhitman@jhmi.edu).

$0022-5223 / \$ 36.00$

Copyright (c) 2016 by The American Association for Thoracic Surgery

http://dx.doi.org/10.1016/j.jtcvs.2015.12.049 factors for pneumonia after cardiac surgery have been identified; however, a composite measure of pneumonia risk would have clinical utility. The purpose of this study was to derive and validate a risk score for postoperative pneumonia in cardiac surgery patients.

\section{PATIENTS AND METHODS}

\section{Study Population}

This was a single-institution series that included adult cardiac surgery patients operated on between January 1, 2005, and December 31, 2012. All cardiac surgery patients were included with the exception of those on extracorporeal membrane oxygenation or those undergoing heart transplantation. The database

Scanning this QR code will take you to the article title page. To view the AATS 2015 Webcast, see the URL at the end of the article. 


\section{Abbreviations and Acronyms \\ $\mathrm{CABG}=$ coronary artery bypass grafting \\ PNA = pneumonia \\ STS $=$ Society of Thoracic Surgeons}

we used was our institutional Society for Thoracic Surgeons (STS) registry in conjunction with individual electronic medical records. The institutional review board granted the study exempt status.

\section{Baseline Characteristics}

Preoperative variables that were evaluated in the study were age, gender, weight, height, body mass index, serum creatinine, and comorbidities including diabetes mellitus, chronic renal insufficiency, dialysis dependence, smoking history, hypertension, cerebrovascular accident, chronic lung disease (none, mild, moderate, severe), peripheral vascular disease, myocardial infarction (including timing), and previous cardiac interventions. Operative variables included cardiogenic shock, operative urgency (elective vs urgent/emergent), type of cardiac operation, ventricular assist device placement, tricuspid procedure, cardiopulmonary bypass time, cross clamp time, intra-aortic balloon pump (including pre-, intra-, or postoperative implantation), and intraoperative blood product transfusion (including type and amount of blood product).

\section{Derivation of Risk Score}

The primary outcome was postoperative pneumonia, which was defined by using Centers for Disease Control and Prevention criteria, with diagnosis aided by chest radiographs, positive sputum cultures, transtracheal fluid, bronchial washings, and clinical findings. ${ }^{5}$ The study population was randomly divided in a 3:1 ratio into training and validation sets. In the training set, univariate logistic regression analysis was conducted to evaluate the association between each of the variables listed in the Baseline Characteristics section and postoperative pneumonia. Those variables associated with pneumonia (exploratory $P<.20$ ) and with less than $10 \%$ missing data were entered into the multivariable model. Continuous variables were initially modeled in a continuous manner in the multivariable model. Those significant as continuous variables were then categorized for the purposes of being able to assign risk points. Optimal thresholds for these significant continuous variables were determined using Lowess smoothing plots to identify linear breakpoints. Significant risk factors in the final multivariable model were then assigned points that corresponded to the relative regression coefficient of that risk factor. For simplicity and in an effort to avoid decimal points, the regression coefficient of the significant risk factor was multiplied by a factor of 10 and rounded to the nearest whole integer. A composite risk score was then generated by summing the individual risk points.

\section{Validation of Risk Score}

The predictive capability of the composite risk score was then evaluated in the validation set. Statistical tests for evaluation included logistic regression and area under receiver operating characteristic curve, or c-index, analysis. Weighted regression analysis was also conducted in the validation set to evaluate the correlation between predicted and actual rates of pneumonia stratified by risk score, with weights assigned to the number of patients with that particular score. The c-index of the composite risk score was also compared to the c-index of the STS prolonged ventilation model in predicting rates of postoperative pneumonia. Continuous data are presented as mean \pm standard deviation, and frequency data as number (percentage), unless otherwise noted. Statistical analyses were performed with version 11 STATA software (Stata Corp LP, College Station, Tex).

\section{RESULTS \\ Baseline Characteristics}

There were 6222 patients included in the study. The average age was 61 years; $66 \%$ of the patients were men (Table 1). The most common comorbidity was hypertension, which was present in $73 \%$. Nearly one-third of patients were diabetic $(27 \%)$, a minority of patients had a history of cerebrovascular disease $(10 \%)$ or dialysis dependence $(4 \%)$, and smokers comprised $17 \%$ of the study population. These baseline patient-related characteristics were comparable between the training $(75 \% ; \mathrm{n}=4666)$ and validation cohorts $(25 \% ; \mathrm{n}=1556)$ (Table 1).

The most commonly performed operations were isolated coronary artery bypass grafting (CABG) $(38 \%)$ or isolated valve procedures $(35 \%$ ) (Table 1 ); $62 \%$ of cases were performed on an elective basis. Intra-aortic balloon pump implantation was required in $9 \%$ of patients, and in most cases these were placed preoperatively. Operative variables were similar between the training and validation sets (Table 1).

\section{Risk Score Derivation}

The overall in-hospital mortality rate was $5.2 \%$ $(\mathrm{n}=326)$, with a $9.7 \%$ rate for nonelective cases and a $2.5 \%$ rate for elective cases $(P<.001)$. A total of 282 $(5 \%)$ patients developed postoperative pneumonia. In the training cohort, predictors of pneumonia in univariate analysis that were included in the multivariable model included age, serum creatinine, hypertension, chronic lung disease, peripheral vascular disease, prior cardiac intervention, myocardial infarction in the past 24 hours, cardiogenic shock, emergent operation, operative type, cardiopulmonary bypass time, aortic cross clamp time, intraoperative red blood cell transfusion, tricuspid valve procedure, and pre- or intraoperative intra-aortic balloon pump placement (Table 2). Significant predictors in the multivariable model included age $\geq 65$ years, chronic lung disease, peripheral vascular disease, cardiopulmonary bypass time $>100$ minutes, intraoperative red blood cell transfusion, and pre- or intraoperative intra-aortic balloon pump implantation. When a parsimonious model was created that included only the significant multivariable predictors, the relative odds ratios and regression coefficients remained similar to the model shown in Table 2.

These variables were assigned varying points relative to their regression coefficients in the multivariable model (Table 3). Overall, there were 33 possible points in the composite risk index, with scores ranging from 0 to 29 in the training cohort with a mean of $5.4 \pm 4.5$. The predicted rate of postoperative pneumonia ranged from $1.2 \%$ for those with a score of 0 , to $59.0 \%$ for those with a score of 33 (Figure 1). The c-index of the model in predicting 
TABLE 1. Comparison of baseline characteristics between the training and validation sets

\begin{tabular}{|c|c|c|c|}
\hline Variable & $\begin{array}{l}\text { Training set } \\
(\mathrm{n}=4666)\end{array}$ & $\begin{array}{c}\text { Validation set } \\
(\mathbf{n}=1556)\end{array}$ & $P$ \\
\hline \multicolumn{4}{|l|}{ Patient } \\
\hline Age (y) & $61.2 \pm 14.9$ & $61.4 \pm 14.7$ & .69 \\
\hline Male $(\%)$ & $3067(66)$ & $1020(66)$ & .90 \\
\hline Body mass index $\left(\mathrm{kg} / \mathrm{m}^{2}\right)$ & $28.8 \pm 7.6$ & $29.0 \pm 9.4$ & .38 \\
\hline Diabetes mellitus (\%) & $1257(27)$ & $404(26)$ & .45 \\
\hline Hypertension (\%) & $3411(73)$ & $1112(71)$ & .21 \\
\hline Dialysis dependence $(\%)$ & $135(4)$ & $38(4)$ & .34 \\
\hline Serum creatinine $(\mathrm{mg} / \mathrm{dL})$ & $1.20 \pm 1.10$ & $1.18 \pm 1.12$ & .56 \\
\hline Cerebrovascular disease (\%) & $467(10)$ & $177(11)$ & .13 \\
\hline Chronic lung disease & & & .32 \\
\hline None $(\%)$ & $4053(87)$ & $1346(87)$ & \\
\hline Mild (\%) & 338 (7) & $131(8)$ & \\
\hline Moderate $(\%)$ & $184(4)$ & $54(3)$ & \\
\hline Severe $(\%)$ & $90(2)$ & $25(2)$ & \\
\hline Peripheral vascular disease (\%) & $473(10)$ & $160(10)$ & .87 \\
\hline Prior cardiac intervention $(\%)$ & $1437(31)$ & $486(31)$ & .75 \\
\hline Cardiogenic shock (\%) & $124(3)$ & $37(2)$ & .55 \\
\hline Myocardial infarction (\%) & $1294(28)$ & 413 (27) & .36 \\
\hline \multicolumn{4}{|l|}{ Operative } \\
\hline Elective case $(\%)$ & $2886(62)$ & $976(63)$ & .54 \\
\hline Type of operation & & & .88 \\
\hline Valve (\%) & $1613(35)$ & $534(34)$ & \\
\hline CABG $(\%)$ & $1776(38)$ & $595(38)$ & \\
\hline Combined valve and CABG $(\%)$ & $487(10)$ & $172(11)$ & \\
\hline Other $(\%)$ & $790(17)$ & $255(16)$ & \\
\hline Ventricular assist device $(\%)$ & $647(28)$ & $213(28)$ & .94 \\
\hline Tricuspid valve procedure $(\%)$ & $180(4)$ & $58(4)$ & .82 \\
\hline $\begin{array}{l}\text { Intraoperative red blood } \\
\text { cell transfusion }(\%)\end{array}$ & $2656(57)$ & $896(58)$ & .66 \\
\hline $\begin{array}{l}\text { Cardiopulmonary bypass } \\
\text { Time (min) }\end{array}$ & $122.1 \pm 53.3$ & $122.1 \pm 55.7$ & .98 \\
\hline Aortic cross clamp time (min) & $78.6 \pm 34.5$ & $77.9 \pm 34.6$ & .52 \\
\hline Intra-aortic balloon pump (\%) & $427(9)$ & $127(8)$ & .24 \\
\hline Intra-aortic balloon pump & & & .83 \\
\hline Preoperative $(\%)$ & $262(61)$ & $79(62)$ & \\
\hline Intraoperative (\%) & $140(33)$ & $39(31)$ & \\
\hline Postoperative (\%) & $25(6)$ & $9(7)$ & \\
\hline
\end{tabular}

$C A B G$, Coronary artery bypass grafting.

pneumonia was 0.72 . The likelihood ratio test $\chi^{2}$ of the model was $116.8(P<.001)$ and the Hosmer-Lemeshow goodness-of-fit test was not significant at $P=.79$, suggesting the model was a good fit.

\section{Risk Score Validation}

Risk scores ranged from 0 to 24 in the validation cohort, with a mean of $5.3 \pm 4.6$. The risk score was predictive of postoperative pneumonia in the validation set (odds ratio $1.21,95 \%$ confidence interval $1.12-1.31 ; P<.001)$. The c-index of the composite score in predicting pneumonia in the validation cohort was 0.76 . There was significant correlation between predicted rates of pneumonia based on the training cohort and actual rates of pneumonia in the validation cohort, stratified by risk score and weighted by number of patients with that particular score $(r=0.74$, $P<.001)$. The composite risk score outperformed the STS model for prolonged ventilation in predicting postoperative pneumonia (c-index 0.76 vs 0.71 ). When stratified by categories of risk score in the training set, patients with a score of 0 had a pneumonia rate of $1.5 \%$, those with a score of 1 to 5 had a pneumonia rate of $2.2 \%$, those with a score of 6 to 10 had a pneumonia rate of $2.6 \%$, and those with a score greater than 10 had a pneumonia rate of $8.5 \%(P<.001)$ (Figure 2$)$. Similar rates were observed in the validation set, with pneumonia rates of $0 \%, 1.6 \%, 2.5 \%$, and $8.2 \%$ with these risk categories, respectively $(P=.001)$.

\section{DISCUSSION \\ Study Rationale}

In a study of more than 4300 cardiac surgical patients, pneumonia represented the most common major hospital-acquired infection, representing $48 \%$ of these complications. ${ }^{1}$ The development of postoperative pneumonia after cardiac surgery is associated with an adverse impact on clinical outcomes and increased costs of care. A meta-analysis of 11 studies found that pneumonia had a substantial effect on increased mortality and increased length of intensive care unit stay in cardiac patients. ${ }^{2}$ A study by our group demonstrated that even after adjusting for other postoperative complications as well patient and operativerelated variables, postoperative pneumonia was associated with a significant increase in inpatient costs of CABG. ${ }^{4}$ Given its not uncommon prevalence and adverse impact on clinical outcomes and costs, it is important to delineate risk factors for pneumonia not only to improve patient outcomes but to potentially provide more cost-effective pathways of delivering care to cardiac surgery patients.

\section{Study Findings}

In this study, we used data from 6222 patients undergoing open heart surgery at our institution to develop and validate a composite risk index for postoperative pneumonia. We constructed a 33-point risk score incorporating 6 pre- and intraoperative predictors of pneumonia. By several measures, the risk score demonstrated predictive capability in evaluating the risk of postoperative pneumonia, and the score was validated as a predictive tool in a patient cohort that was completely external and separate from the training cohort. We believe the score is particularly useful in that it predicts a wide range of pneumonia risk $(1.2 \%-59 \%)$ and is easily calculable at the bedside.

\section{Clinical Utility of the Risk Score}

We believe the potential clinical utility of this risk score lies in risk modification, directing diagnostic and 
TABLE 2. Multivariable logistic regression model for postoperative pneumonia

\begin{tabular}{|c|c|c|c|}
\hline Covariates & $\begin{array}{c}\text { Odds ratio } \\
(95 \% \text { confidence interval })\end{array}$ & $\begin{array}{c}\text { Regression coefficient } \\
(95 \% \text { confidence interval })\end{array}$ & $\boldsymbol{P}$ \\
\hline Age $\geq 65 y^{*}$ & $1.40(1.01-1.94)$ & $0.33(0.01-0.66)$ & .04 \\
\hline Hypertension & $1.08(0.72-1.62)$ & $0.08(-0.33$ to 0.48$)$ & .71 \\
\hline \multicolumn{4}{|l|}{ Chronic lung disease } \\
\hline None & Reference & Reference & Ref. \\
\hline Mild & $1.97(1.22-3.16)$ & $0.68(0.20-1.15)$ & .005 \\
\hline Moderate & $3.07(1.80-5.23)$ & $1.12(0.59-1.65)$ & $<.001$ \\
\hline Severe & $3.31(1.56-7.03)$ & $1.20(0.45-1.95)$ & .002 \\
\hline Peripheral vascular disease & $1.69(1.11-2.56)$ & $0.52(0.11-0.94)$ & .01 \\
\hline Prior cardiac intervention & $1.04(0.74-1.47)$ & $0.04(-0.30$ to 0.38$)$ & .81 \\
\hline Myocardial infarction in past $24 \mathrm{~h}$ & $2.40(0.66-8.73)$ & $0.88(-0.41$ to 2.17$)$ & .18 \\
\hline Cardiogenic shock & $1.04(0.46-2.34)$ & $0.04(-0.77$ to 0.85$)$ & .93 \\
\hline Serum creatinine (increasing, per $1 \mathrm{mg} / \mathrm{dL}$ ) & $1.00(0.87-1.15)$ & $-0.001(-0.14$ to 0.14$)$ & .98 \\
\hline Emergent operation & $1.28(0.92-1.78)$ & $0.25(-0.08$ to 0.58$)$ & .14 \\
\hline \multicolumn{4}{|l|}{ Type of operation } \\
\hline Valve & Reference & Reference & Ref. \\
\hline CABG & $1.25(0.81-1.91)$ & $0.22(-0.21$ to 0.65$)$ & .31 \\
\hline Valve + CABG & $1.48(0.92-2.37)$ & $0.39(-0.08$ to 0.86$)$ & .10 \\
\hline Other & $1.43(0.74-2.76)$ & $0.36(-0.30$ to 1.02$)$ & .28 \\
\hline Cardiopulmonary bypass time $>100 \mathrm{~min}^{*}$ & $1.71(1.13-2.61)$ & $0.54(0.12$ to 0.96$)$ & .01 \\
\hline Aortic cross clamp time (increasing, per $1 \mathrm{~min}$ ) & $1.00(0.99-1.00)$ & $0.00(-0.006$ to 0.005$)$ & .91 \\
\hline Intraoperative red blood cell transfusion & $1.08(1.03-1.13)$ & $0.08(0.03-0.13)$ & .002 \\
\hline Tricuspid valve procedure & $1.73(0.89-3.36)$ & $0.55(-0.12$ to 1.21$)$ & .11 \\
\hline Pre- or intraoperative intra-aortic balloon pump implantation & $2.01(1.30-3.11)$ & $0.70(0.26-1.13)$ & .002 \\
\hline
\end{tabular}

$C A B G$, Coronary artery bypass grafting. *Age and cardiopulmonary bypass time were also significant predictors of postoperative pneumonia when modeled as continuous variables.

preventative measures, and in clinical research. With respect to risk modification, we would propose that preventing or optimizing management of chronic lung disease and peripheral vascular disease, both of which were risk factors in our analysis, could potentially be met with a reduction in rates of postoperative pneumonia. As increasing severity of chronic lung disease was accompanied by an increasing number of risk points in our analysis, perhaps initiating a program of respiratory physiotherapy or medically optimizing patients with chronic lung disease preoperatively would be prudent. A randomized clinical trial published in 2006 demonstrated that preoperative inspiratory muscle

TABLE 3. Composite risk score for postoperative pneumonia

\begin{tabular}{lc}
\hline \multicolumn{1}{c}{ Risk factor } & Points \\
\hline Age $\geq 65$ y & 3 \\
Chronic lung disease & \\
$\quad$ None & 0 \\
Mild & 7 \\
Moderate & 11 \\
Severe & 12 \\
Peripheral vascular disease & 5 \\
Cardiopulmonary bypass time $>100$ min & 5 \\
Intraoperative red blood cell transfusion & 1 \\
Pre- or intraoperative intra-aortic balloon pump & 7 \\
Total points possible & 33 \\
\hline
\end{tabular}

training reduced rates of postoperative pneumonia in high-risk cardiac surgery patients from $16.1 \%$ to $6.5 \%$, which was statistically significant. ${ }^{6}$ Similarly, medical optimization with aspirin and statin therapy, as well as diet and exercise modifications, may be useful in lessening the burden of peripheral vascular disease preoperatively, which may in turn lead to improved ambulatory status and lower rates of pneumonia following cardiac surgery.

Another risk factor identified in our analysis that may be modifiable is intraoperative red blood cell transfusion. Although blood transfusions can be lifesaving, there is an

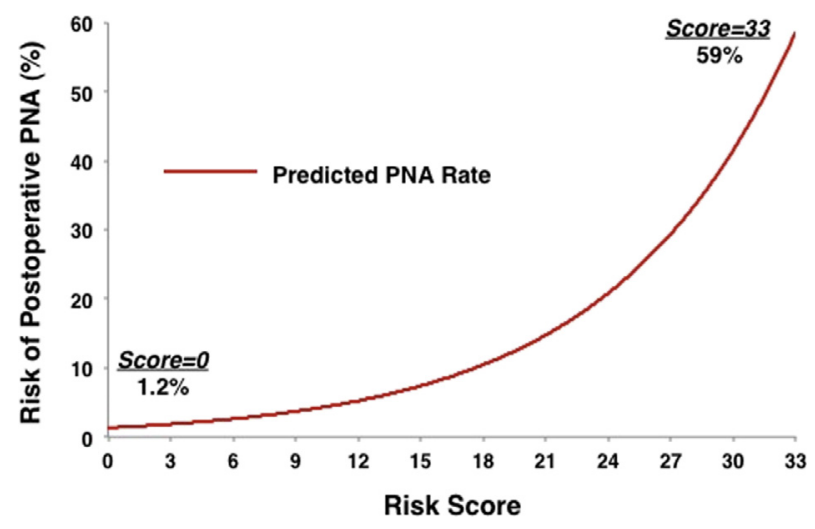

FIGURE 1. Predicted rates of postoperative pneumonia based on risk score as derived from the training cohort. PNA, Pneumonia. 


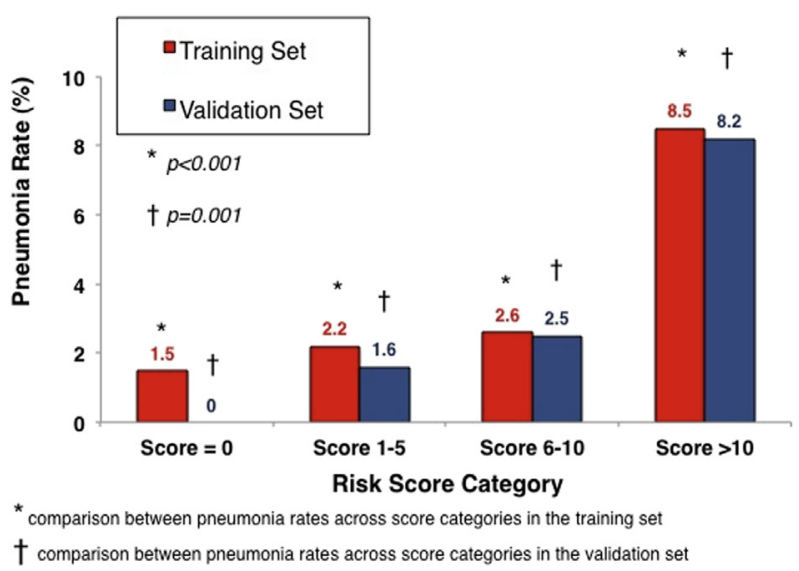

FIGURE 2. Rates of pneumonia by categories of risk score in the training and validation sets.

increasing awareness in cardiac surgery that blood transfusions have adverse clinical effects as well. ${ }^{7}$ A multi-institutional study that included 284 intensive care units in the United States found that blood transfusions independently increased the odds of ventilator-associated pneumonia. ${ }^{8}$ Another analysis of more than 34,000 patients with CABG found that even after matching for predicted risk of mortality, patients receiving a transfusion were more likely to die from infectious or pulmonary complications than those patients not receiving a transfusion. ${ }^{9}$ This link between transfusions and pneumonia risk may in part be explained by increasing evidence that transfusions are immunomodulatory and suppress the host immune system, thereby increasing susceptibility to infectious complications. ${ }^{10,11}$ STS clinical practice guidelines provide specific recommendations for blood conservation given the increasing awareness that blood transfusions are not benign but rather associated with adverse effects, and these techniques for blood conservation when applied may be met with decreased rates of postoperative pneumonia. $^{12,13}$

Another implication of this risk score may be in targeting high-risk patient subsets for preventive measures and for clinical research. A previous study found that by implementing sequential measures, including a specific training program, aspiration of subglottic secretions, introduction of an inclinometer, and oral care with chlorhexidine, rates of ventilator-associated pneumonia were reduced significantly. ${ }^{14}$ Future studies that aim to evaluate the clinical benefit of specific interventions on reducing pneumonia risk may benefit from focusing on high-risk patients who could be identified by using the risk score. As patients with low-risk scores have low pneumonia rates, it may not be very cost-effective to include such patients in these studies. Instead, by focusing on patients at higher risk for pneumonia, the interventions may be more cost-effective when extrapolated to larger populations.

\section{Previous Studies}

A previous multi-institutional study evaluated more than 160,000 patients undergoing major noncardiac surgery and found that age, chronic obstructive pulmonary disease, and transfusion were associated with pneumonia risk, similar to our results. ${ }^{15}$ There were other additional risk factors identified, including functional status, weight loss, and cerebrovascular disease, that were not included in our score. Support with an intra-aortic balloon pump was assigned 7 points in our risk index. A study of cardiac surgery patients requiring intra-aortic balloon pumps found that in patients weaned off support, ventilator-associated pneumonia was the most common cause of death. ${ }^{16}$ Cardiopulmonary bypass induces a systemic inflammatory response that can lead to organ dysfunction, including pulmonary dysfunction, which can manifest as decreased pulmonary compliance, rise in shunt fraction, and increased likelihood of atelectasis and pneumonia. ${ }^{17}$ Not surprisingly, therefore, prolonged cardiopulmonary bypass time was associated with increased pneumonia risk in our analysis.

Another single-center cohort study involving 5582 cardiac surgery patients demonstrated results similar to our analysis. ${ }^{18}$ Moreover, a postoperative pneumonia rate of $3.1 \%$ was observed, with age, chronic obstructive pulmonary disease, preoperative left ventricular ejection fraction, and the interaction between red blood cell transfusion and duration of cardiopulmonary bypass time being identified as significant multivariate predictors of pneumonia. An analysis of the American College of Surgeons' National Surgical Quality Improvement Program registry found that patients experiencing postoperative pneumonia had a more than 10-fold increased rate of 30-day mortality, and predictors of postoperative pneumonia included age, American Society of Anesthesiologists class, chronic obstructive pulmonary disease, dependent functional status, preoperative sepsis, smoking, and type of operation. ${ }^{19}$

\section{Limitations}

A limitation of this study was that some variables that may impact rates of postoperative pneumonia, such as incision type and ejection fraction, were not available in our database. In addition, although there were predefined criteria in our study, there is some inherent degree of variability and subjectivity in the clinical diagnosis of pneumonia. We did not evaluate the severity of the pneumonia, its impact on other clinical outcomes, or its impact on costs of care, although these additional outcomes are certainly of interest. Our study is based on the experience of a single institution, which may limit generalizability to other centers. Finally, our analysis was limited to inpatient outcomes and did not evaluate longitudinal outcomes. Moreover, a subset of postoperative pneumonias develop or are identified after discharge from the index admission 
and our registry did not capture such cases that were diagnosed during readmission or on an outpatient basis.

\section{CONCLUSIONS}

This study developed and validated a 33-point risk score for postoperative pneumonia following cardiac surgery using 6 risk factors. We believe the risk score has clinical utility in that it is easy to calculate at the bedside and predicts a wide range of pneumonia risk. Its potential clinical applications include risk modification and targeted interventions to reduce rates of pneumonia in higher-risk patients. Additionally, future clinical studies could use the risk score for stratification when comparing various patient subsets or therapies.

\section{Conflict of Interest Statement}

Authors have nothing to disclose with regard to commercial support.

You can watch a Webcast of this AATS meeting presentation by going to: http://webcast.aats.org/2015/Video/ Monday/04-27-15_608_1600_Kilic.mp4.

\section{References}

1. Greco G, Shi W, Michler RE, Meltzer DO, Ailawadi G, Hohmann SF, et al. Costs associated with health care-associated infections in cardiac surgery. $J$ Am Coll Cardiol. 2015;65:15-23.

2. He S, Chen B, Li W, Yan J, Chen L, Wang X, et al. Ventilator-associated pneumonia after cardiac surgery: a meta-analysis and systematic review. $J$ Thorac Cardiovasc Surg. 2014;148:3148-55.

3. Brown PP, Kugelmass AD, Cohen DJ, Reynolds MR, Culler SD, Dee AD, et al. The frequency and cost of complications associated with coronary artery bypass grafting surgery: results from the United States Medicare program. Ann Thorac Surg. 2008;85:1980-6.

4. Kilic A, Shah AS, Conte JV, Mandal K, Baumgartner WA, Cameron DE, et al. Understanding variability in hospital-specific costs of coronary artery bypass grafting represents an opportunity for standardizing care and improving resource use. J Thorac Cardiovasc Surg. 2014;147:109-15.

5. Horan TC, Andrus M, Dudeck MA. CDC/NHSN surveillance definition of health care-associated infection and criteria for specific types of infections in the acute care setting. Am J Infect Control. 2008;36:309-32.

6. Hulzebos EH, Helders PJ, Favie NJ, De Bie RA, Brutel de la Riviere A, Van Meeteren NL. Preoperative intensive inspiratory muscle training to prevent postoperative pulmonary complications in high-risk patients undergoing CABG surgery: a randomized clinical trial. JAMA. 2006;296:1851-7.

7. Kilic A, Whitman GJ. Blood transfusions in cardiac surgery: indications, risks, and conservation strategies. Ann Thorac Surg. 2014;97:726-34.

8. Shorr AF, Duh MS, Kelly KM, Kollef MH; CRIT Study Group. Red blood cell transfusion and ventilator-associated pneumonia: a potential link? Crit Care Med. 2004;32:666-74.

9. Paone G, Herbert MA, Theurer PF, Bell GF, Williams JK, Shannon FL, et al. Red blood cells and mortality after coronary artery bypass graft surgery: an analysis of 672 operative deaths. Ann Thorac Surg. 2015;99:1589-90.

10. Opelz G, Vanrenterghem Y, Kirste G, Gray DW, Horsburgh T, Lachance JG, et al. Prospective evaluation of pretransplant blood transfusions in cadaver kidney recipients. Transplantation. 1997;63:964-7.

11. Blumberg N, Heal JM. Effects of transfusion on immune function. Cancer recurrence and infection. Arch Pathol Lab Med. 1994;118:371-9.

12. Ferraris VA, Ferraris SP, Saha SP, Hessel EA II, Haan CK, Royston BD, et al. Perioperative blood transfusion and blood conservation in cardiac surgery: the Society of Thoracic Surgeons and The Society of Cardiovascular Anesthesiologists clinical practice guidelines. Ann Thorac Surg. 2007;83: S27-86.
13. Ferraris VA, Brown JR, Despotis GJ, Hammon JW, Reece TB, Saha SP, et al. 2011 update to the Society of Thoracic Surgeons and the Society of Cardiovascular Anesthesiologists blood conservation clinical practice guidelines. Ann Thorac Surg. 2011;91:944-82.

14. Perez-Granda MJ, Barrio JM, Munoz P, Hortal J, Rincon C, Bouza E. Impact of four sequential measures on the prevention of ventilator-associated pneumonia in cardiac surgery patients. Crit Care. 2014;18:R53.

15. Arozullah AM, Khuri SF, Henderson WG, Daley J. Development and validation of a multifactorial risk index for predicting postoperative pneumonia after major noncardiac surgery. Ann Intern Med. 2001;135:847-57.

16. Castelli P, Condemi A, Munari M, Savi C, Carro C, Vanelli P. Intra-aortic balloon counterpulsation: outcome in cardiac surgical patients. J Cardiothorac Vasc Anesth. 2001;15:700-3.

17. Li S, Price R, Phiroz D, Swan K, Crane TA. Systemic inflammatory response during cardiopulmonary bypass and strategies. J Extra Corpor Technol. 2005; 37:180-8.

18. Allou N, Bronchard R, Guglielminotti J, Dilly MP, Provenchere S, Lucet JC, et al. Risk factors for postoperative pneumonia after cardiac surgery and development of a preoperative risk score. Crit Care Med. 2014;42:1150-6.

19. Gupta H, Gupta PK, Schuller D, Fang X, Miller WJ, Modrykamien A, et al. Development and validation of a risk calculator for predicting postoperative pneumonia. Mayo Clin Proc. 2013;88:1241-9.

Key Words: pneumonia, risk score, outcomes, postoperative complications

\section{Discussion}

Dr E. Blackstone (Cleveland, Ohio). Thank you and the Association for the privilege of studying your paper on this vitally important topic of postoperative hospitalacquired pneumonia.

My questions are informed by the NHLBI-sponsored Cardiothoracic Surgical Trials Network study of postcardiac surgery infections up to 60 days after discharge. As you mentioned, far and away the most common infection was hospital-acquired pneumonia, identified according to CDC definitions and adjudicated by a panel of infectious disease experts.

Question 1: Should you look beyond initial hospitalization for infections? Nearly half the infections in the Network study occurred in the 60 days after hospital discharge, although the percentage was somewhat smaller for pneumonia.

Related question 2: Have you assessed management practices that may be associated with pneumonia? The Network study dug deeply into potentially modifiable management practices associated with infection. Your less-than-robust $\mathrm{C}$ statistic suggests that there is more going on than you can find with standard STS variables alone. I could imagine that a deeper dive may transform your study into a practical quality-improvement initiative.

Finally, while you think about those 2 questions, a comment related to how you developed and validated your score. Your manuscript notes that the score underestimated events in the validation group. The mistake you made is a common one that has been pointed out repeatedly in the statistical literature: odds ratios multiply, not add. At low probabilities, multiplication and addition 
produce deceptively similar results. At higher probabilities, results diverge widely.

Nearly 40 years after logistic regression was introduced, software to do it easily has proliferated, but understanding of the underlying basic math has been lost. Lost also, it seems, is the extensive suite of model validation methods, such as provided in $\mathrm{R}$ software by Frank Harrell at Vanderbilt. Hopkins has some expert biostatisticians that could help you in this regard. Thank you.

Dr Magruder. Thank you for your comments. I think both of your points are excellent, and I have to admit that my response to neither of them will be satisfactory.

To the first point, as far as our limitation and diagnoses to being in the hospital, unfortunately, we don't have more information contained in the STS database. As you know, with any risk score, one of the most important parts to deriving your risk score is that you have to have quite a large number of patients, and the benefit of using STS is that we have tremendous numbers of patients to look at. The down side is that we really have no way to get at those patients who might be diagnosed down the line.

Now, in Maryland, there actually is some new readmission data for hospitals across the state that's coming online, and so we'll take a look and see if we can't maybe use some of that and at least pick up patients who might develop a pneumonia outside of the hospital that is significant enough to get readmitted elsewhere. So that's one thought, and especially because we get patients referred, we might pick up some of those patients.

As to the second point, management strategies, we have not looked at those. We aimed to derive a risk score that we would be able to use to predict a patient's pneumonia risk as soon as the patient arrived to the ICU, which is why we didn't look at some of the extra factors. For example, we didn't look at prolonged intubation or the other factors that we all know drive up pneumonia risk. Though you're right, that would be an excellent thing to look at.

Dr K. Khargi (Maastricht, The Netherlands). I'm missing actually factors like the procedure double mammary, bilateral mammary artery, the preoperative psychological situation of the patients, the ability to comply with physiotherapy, the ability to start his physical exercise. I think those are factors that are really very important, but they didn't show up here in your study. How come?

Dr Magruder. So that's a good question. Again, because we're looking at STS data, some of those things simply aren't captured as a variable. For example, the ability to participate in incentive spirometry, those kinds of pulmonary toilet activities, that's something that's difficult to capture. The only variable we have in terms of preoperative pulmonary status is a variable that is not even a diagnosis of COPD. It's the STS's chronic lung disease variable that has some loose definitions.

As far as some of the other variables, so we did not look at bilateral internal mammary usage, but that would be a good one. We did look at operative type, another procedure type. And additionally, as a proxy for the difficulty of operation, we did look at bypass time, and that was a significant variable in our model.

So in short, I think those are all excellent points, and I agree with those. Unfortunately, we're limited in some of the aspects that we all know undoubtedly influence the risk of postoperative pneumonia but that we're simply not able to capture in our 5 or 6000 patients. 